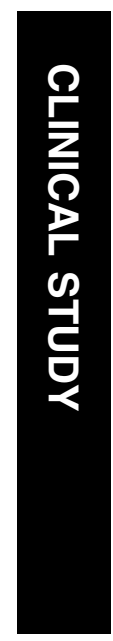

\title{
Inter-device reproducibility of the scanning laser polarimeter with variable cornea compensation
}

${ }^{1}$ Department of Ophthalmology, Hadassah University Hospital Jerusalem, Israel

${ }^{2}$ Hebrew UniversityHadassah Medical School Jerusalem, Israel

Correspondence:

EZ Blumenthal

Tel: + 97226776580

Fax: +97226758926

E-mail: eblumenthal@

md.huji.ac.il

Received: 6 July 2003

Accepted in revised form: 19 January 2004

Published online: 30 July 2004

Presented in part at the Association for Research in Vision and Ophthalmology (ARVO) meeting, Fort Lauderdale, Florida, May 2003.

\begin{abstract}
Objective To evaluate the interdevice reproducibility of retinal nerve fibre layer (RNFL) thickness measurements obtained with the commercially available GDx-VCC, a scanning laser polarimeter with variable (individualized) corneal compensation. Methods A prospective instrument validation study in which 13 GDx-VCC devices were tested. One eye each, from three normal subjects were used to test each of the devices, on the same day, by an experienced operator. Variability and reproducibility for each of five GDx parameters were calculated. Results For each of five tested GDx parameters, the coefficient of variation and 95\% confidence interval range $(\mu \mathrm{m})$, for the 13 devices, respectively, were: TSNIT avg: $5.1 \%$, $3.84 \mu \mathrm{m}$; Superior avg: $5.3 \%, 4.82 \mu \mathrm{m}$; Inferior avg: $6.1 \%, 5.50 \mu \mathrm{m}$; TSNIT standard deviation: $8.6 \%, 2.92 \mu \mathrm{m}$; and nerve fibre indicator (NFI): N/A, 5.69. Item reliability (Cronbach's alpha) for the five GDx parameters are: TSNIT-Avg: 0.97, Sup-Avg: 1.00, Inf-Avg: 0.84, TSNIT-SD: 0.99, NFI: 0.99 .
\end{abstract}

Conclusions With the commercially available GDx-VCC, our results indicate that RNFL measurements appear reproducible across devices.

Eye (2005) 19, 308-311. doi:10.1038/sj.eye.6701472 Published online 30 July 2004

Keywords: glaucoma; retinal nerve fibre layer; imaging; scanning laser polarimetry

\section{Introduction}

Assessment of the retinal nerve fibre layer (RNFL) is an important modality in diagnosing glaucoma and following patients over time. ${ }^{1}$
Quantifying RNFL thickness at the peripapillary region holds promise in providing information on the structural damage occurring in glaucoma. To this end, several technologies exist for measuring RNFL thickness, each utilizing different properties of light. ${ }^{2}$

Scanning laser polarimetry (SLP) combines a confocal scanning laser ophthalmoscope coupled with an integrated polarimeter, providing a quantitative evaluation of the RNFL thickness. ${ }^{3}$ Polarized light undergoes a phase shift as it passes through any polarizing medium, a shift that is proportional to both the polarizing properties as well as the thickness of that medium. In the eye, in addition to the RNFL, both the cornea ${ }^{4-7}$ and, to a lesser extent, the lens ${ }^{8}$ are polarizing tissues. This is due to the tightly packed parallel infrastructure makeup of these tissues.

Recently, it became evident that for meaningful SLP data, it is necessary to neutralize the anterior segment polarization component in order to measure the RNFL contribution reliably. ${ }^{5,9,10}$ The magnitude and axis of the corneal component has been shown to differ widely among different individuals. ${ }^{4,5}$ RNFL polarimetry employing the custom corneal compensation correction relies on a twostep approach: first, a foveal scan is taken. Any retardation measured is attributed to the cornea (and lens), as the foveal region is known to be devoid of axons, and thus presumed to be neutral with respect to polarization properties. Once the corneal contribution of that given eye is ascertained and neutralized, a second scan of the peripapillary region is taken.

In this study, we analysed interdevice reproducibility of the commercially available GDx-VCC instrument. Inter-device reproducibility is an important component of 
variability that is often overlooked, perhaps owing to the difficulty of executing a study in which multiple devices need to be available simultaneously. In fact, interdevice reproducibility is what makes the results of one study applicable to the clinical setting of others. Hence, the lack of interdevice reproducibility would significantly weaken the generalizability of any data published.

\section{Methods}

\section{Subjects}

In all, 13 GDx-VCC devices were evaluated using one randomly selected eye from three normal volunteers aged 38, 39, and 46 years, two men, and a woman. Informed consent was obtained from all participants and Institutional Ethics Committee approval was obtained. Two subjects underwent a complete eye examination, including a medical history, slit-lamp examination, intraocular pressure measurement, and a standard fullthreshold visual field. They were classified as normal, based on: intraocular pressure measurement $<21 \mathrm{mmHg}$ and no documented prior elevated measurements, normal-appearing optic discs, as judged by a glaucomatrained ophthalmologist, and a visual field glaucoma hemifield test within normal limits. The third subject did not undergo an eye examination and is hence presumed as normal, based on a negative medical history and a normal GDx-VCC examination $(\mathrm{NFI}=11)$.

\section{The GDx-VCC instrument}

The GDx-VCC (Laser Diagnostics Technologies, San Diego, CA USA) is the first commercially available variable cornea compensator scanning laser polarimeter. It differs from previous models, including the GDx with a fixed compensator, ${ }^{11}$ the Access model (also with a fixed compensator), which it resembles externally, as well as from the modified GDx mounted with a manual variable corneal compensator, ${ }^{4,5,10}$ a noncommercial investigational (prototype) model, of which only a few units were ever produced. For this study, we used 13 GDx-VCC units set up side by side.

\section{Measurements}

A single experienced examiner scanned each of the three tested eyes during a single day, on all 13 machines. Two examiners participated in the study, such that one examiner scanned two of the subjects. The order in which machines were used was random. Each scan, on each machine, was composed of a foveal scan (also referred to as a corneal scan), and a peripapillary scan. Each examiner was instructed to achieve a high quality scan, as judged by her experience, as well as a quality score of at least 8/10 (if achievable), yet not to re-scan beyond three attempts. Once a good-quality scan was obtained, a printed copy was made and the examiner and examinee proceeded to the next machine.

\section{GDx parameters}

From the 16 parameters appearing on the GDx printout (software version: GDx-VCC 5.1.0), we chose the five parameters appearing on the first printout page, which are those parameters chosen by the manufacturer to be the most informative and that are best able to discriminate between normal and glaucomatous eyes. These parameters are: TSNIT average (the average RNFL thickness within the measured ring, named 'TemporalSuperior-Nasal-Inferior-Temporal'), Superior average (the average of the RNFL thickness values of the points within the ring along the superior $120^{\circ}$ section), Inferior average (the average of the RNFL thickness values of the points within the ring along the inferior $120^{\circ}$ section), TSNIT standard deviation (the standard deviation of the data points within the measured ring), and NFI, which is a support vector machine-derived parameter indicating the likelihood that the eye is glaucomatous.

\section{Statistical analysis}

Data were analysed using JMP statistical software (SAS Institute, Cary, NC, USA) Coefficient of variation, 95\% confidence intervals (95\% CI), and Cronbach's alpha reliability were calculated for each of the five GDx parameters.

\section{Results}

All scans were subjectively judged to be of good quality by the experienced examiners. Of the 39 scans, all received a machine-determined quality score of 8-9 (out of 10), apart from three scans that scored 7.

\section{Interdevice reproducibility}

Interdevice variability data for each eye, as well as averaged across eyes, are presented in Table 1. A major question underlying this study was to ascertain whether all 13 devices provide data that are comparable, or perhaps some of the devices generate data that are significantly different. To test for this, we calculated the item reliability (Cronbach's alpha) for each of the five GDx parameters, comparing across devices (Table 2). A high reliability (0.97-1.00) was found between devices for four of the five parameters, while the Inferior-Average parameter showed somewhat lesser reliability (0.84). 
Table 1 Interdevice variability data for five GDx parameters

\begin{tabular}{|c|c|c|c|c|c|c|c|c|}
\hline \multirow[t]{2}{*}{ Subjects } & \multicolumn{2}{|c|}{$A$} & \multicolumn{2}{|c|}{$B$} & \multicolumn{2}{|c|}{ C } & \multicolumn{2}{|c|}{ Average } \\
\hline & $\mathrm{COV}$ & $95 \% \mathrm{CI}$ & $\mathrm{COV}$ & $95 \% \mathrm{CI}$ & $\mathrm{COV}$ & $95 \% C I$ & $\mathrm{COV}$ & $95 \% \mathrm{CI}$ \\
\hline TSNIT-Avg & 4.22 & 3.19 & 6.75 & 4.93 & 4.22 & 3.40 & 5.06 & 3.84 \\
\hline SUP-Avg & 3.92 & 3.25 & 7.23 & 6.08 & 4.64 & 5.12 & 5.27 & 4.82 \\
\hline INF-Avg & 4.11 & 3.69 & 9.03 & 8.01 & 5.09 & 4.82 & 6.08 & 5.50 \\
\hline TSNIT-SD & 7.65 & 1.75 & 16.37 & 4.39 & 1.89 & 2.63 & 8.63 & 2.92 \\
\hline NFI & 31.37 & 4.32 & 23.16 & 7.69 & 92.44 & 5.07 & 48.99 & 5.69 \\
\hline
\end{tabular}

The coefficient of variation (COV) is given as a percentage. The 95\% CI for the mean (95\% CI) are given as a range in microns ( $\mu \mathrm{m})$ for the averages of the TSNIT, superior (SUP), and inferior (INF), parameters and in absolute values for the TSNIT-SD and the NFI parameters. NFI, nerve fibre indicator; Avg. average.

Table 2 Item reliability (Cronbach's alpha) for each of the five GDx parameters

\begin{tabular}{lccccc}
\hline & TSNIT-Avg & Sup-Avg & Inf-Avg & TSNIT-SD & NFI \\
\hline $\begin{array}{l}\text { Cronbach's } \\
\text { alpha }\end{array}$ & 0.97 & 1.00 & 0.84 & 0.99 & 0.99 \\
\hline
\end{tabular}

Figure 1 presents the distribution of data across devices by subjects for the TSNIT-Average parameter.

\section{Discussion}

Interdevice reproducibility is one component of reproducibility, alongside interexaminer, intrasession, intersession, as well as short- and long-term variability. All are important in the process of evaluating any new diagnostic technology. It would be beneficial to have a model eye that could be used periodically to test, calibrate, and consequently standardize an individual machine against a gold standard. Such a model eye exists, for instance, for standardized ophthalmic echography. ${ }^{12}$ However, in the absence of such a model eye for scanning laser polarimetry, we chose to submit a human eye to the test, presuming that any changes observed during the course of several hours, in a normal eye, should be attributed to interdevice variability rather than to true anatomical changes in the RNFL. In this interdevice reproducibility study, the 'tested subjects' were in fact the GDx devices $(n=13)$.

Interdevice reproducibility data are somewhat difficult to collect, owing to the need to assemble a large number of instruments. The only study on interdevice reproducibility on any RNFL-imaging device that we are familiar with is a study by Colen et $a l^{13}$ performed using the previous version of the GDx (without the variable corneal compensator) and included a subanalysis of three GDx devices. In contrast, comprehensive intradevice reproducibility data exist for the different imaging devices. ${ }^{11,13-18}$

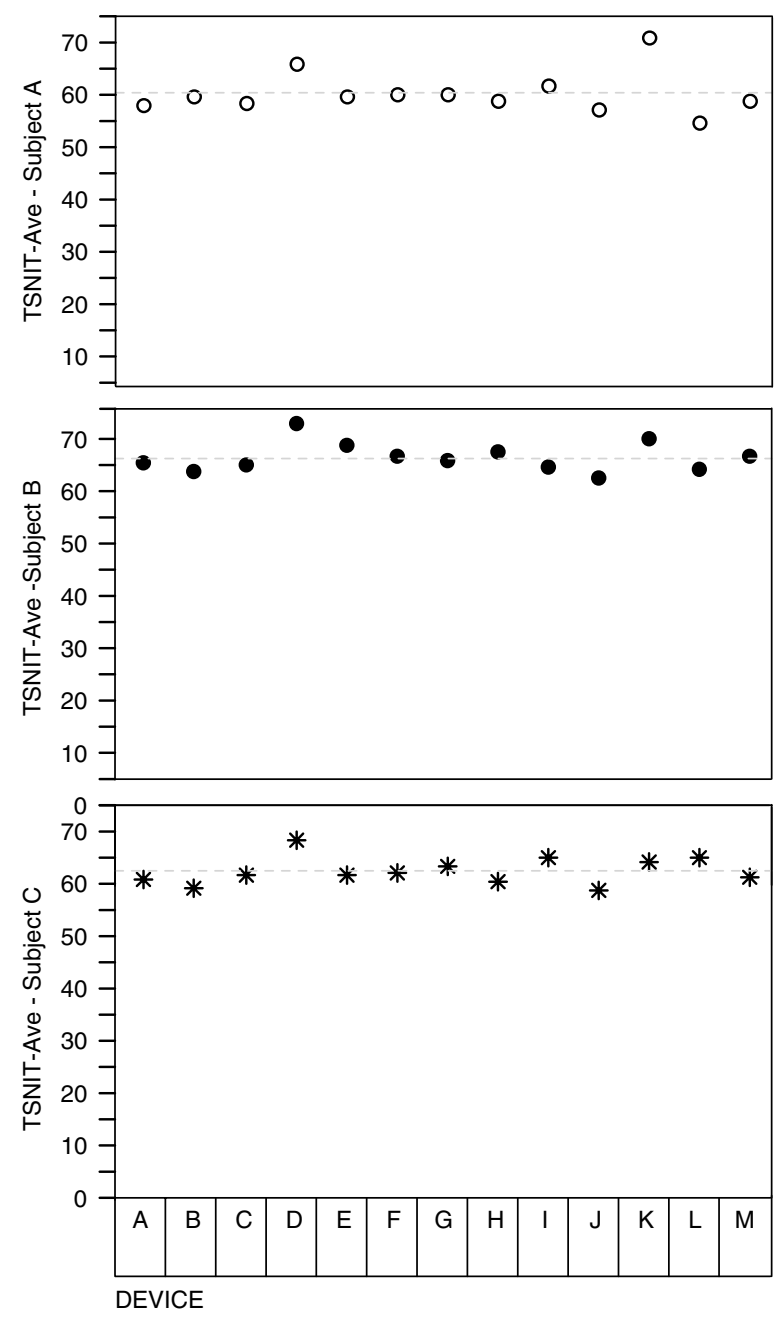

Figure 1 TSNIT average plotted for each of the 13 devices, showing the between-device distribution of measurements for each subject. For each subject, the mean for all devices is presented as a horizontal dotted line.

Subject $\mathrm{C}$ did not undergo a full eye examination and visual field, and is hence only presumed to be normal. As glaucomatous eyes have previously demonstrated higher 
variability in structural measurements, ${ }^{13,15}$ inadvertently including a non-normal eye, if anything, would have increased the variability found in this study.

In this study, we examined the interdevice reproducibility of $13 \mathrm{GDx}-\mathrm{VCC}$ devices. Both COV and 95\% CI range $(5.06 \%$ and $3.84 \mu \mathrm{m}$, respectively) for the TSNIT average RNFL thickness parameter combined with the very high reliability alpha suggest good reproducibility across devices. In conclusion, we found the interdevice reproducibility to be acceptable for comparing data across devices.

\section{References}

1 Jonas JB, Dichtl A. Evaluation of the retinal nerve fiber layer. Surv Ophthalmol 1996; 40: 369-378.

2 Blumenthal EZ, Weinreb RN. Assessment of the retinal nerve fiber layer in clinical trials of glaucoma neuroprotection. Surv Ophthalmol 2001; 45(Suppl 3): S305-S312.

3 Weinreb RN, Dreher AW, Coleman A, Quigley H, Shaw B, Reiter K. Histopathologic validation of Fourier-ellipsometry measurements of retinal nerve fiber layer thickness. Arch Ophthalmol 1990; 108: 557-560.

4 Greenfield DS, Knighton RW, Huang XR. Effect of corneal polarization axis on assessment of retinal nerve fiber layer thickness by scanning laser polarimetry. Am J Ophthalmol 2000; 129: 715-722.

5 Weinreb RN, Bowd C, Greenfield DS, Zangwill LM. Measurement of the magnitude and axis of corneal polarization with scanning laser polarimetry. Arch Ophthalmol 2002; 120: 901-906.

6 Greenfield DS, Knighton RW, Feuer WJ, Schiffman JC, Zangwill L, Weinreb RN. Correction for corneal polarization axis improves the discriminating power of scanning laser polarimetry. Am J Ophthalmol 2002; 134: 27-33.

7 Gurses-Ozden R, Pons ME, Barbieri C, Ishikawa H, Buxton $\mathrm{DF}$, Liebmann JM et al. Scanning laser polarimetry measurements after laser-assisted in situ keratomileusis. Am J Ophthalmol 2000; 129: 461-464.

8 Park RJ, Chen PP, Karyampudi P, Mills RP, Harrison DA, Kim J. Effects of cataract extraction with intraocular lens placement on scanning laser polarimetry of the peripapillary nerve fiber layer. Am J Ophthalmol 2001; 132 507-511.

9 Garway-Heath DF, Greaney MJ, Caprioli J. Correction for the erroneous compensation of anterior segment birefringence with the scanning laser polarimeter for glaucoma diagnosis. Invest Ophthalmol Vis Sci 2002; 43: 1465-1474.

10 Zhou Q, Weinreb RN. Individualized compensation of anterior segment birefringence during scanning laser polarimetry. Invest Ophthalmol Vis Sci 2002; 43: 2221-2228.

11 Kook MS, Sung K, Park RH, Kim KR, Kim ST, Kang W. Reproducibility of scanning laser polarimetry (GDx) of peripapillary retinal nerve fiber layer thickness in normal subjects. Graefes Arch Clin Exp Ophthalmol 2001; 239: 118-121.

12 Ossoinig KC. Standardized echography: basic principles, clinical applications, and results. Int Ophthalmol Clin 1979; 19: $127-210$.

13 Colen TP, Tjon-Fo-sang MJ, Mulder PG, Lemij HG. Reproducibility of measurements with the nerve fiber analyzer (NFA/GDx). J Glaucoma 2000; 9: 363-370.

14 Janknecht P, Funk J. Optic nerve head analyser and Heidelberg retina tomograph: accuracy and reproducibility of topographic measurements in a model eye and in volunteers. Br J Ophthalmol 1994; 78: 760-768.

15 Blumenthal EZ, Williams JM, Weinreb RN, Girkin CA, Berry CC, Zangwill LM. Reproducibility of nerve fiber layer thickness measurements by use of optical coherence tomography. Ophthalmology 2000; 107: 2278-2282.

16 Jones AL, Sheen NJ, North RV, Morgan JE. The Humphrey optical coherence tomography scanner: quantitative analysis and reproducibility study of the normal human retinal nerve fibre layer. Br J Ophthalmol 2001; 85: 673-677.

17 Miglior S, Albe E, Guareschi M, Rossetti L, Orzalesi N. Intraobserver and interobserver reproducibility in the evaluation of optic disc stereometric parameters by Heidelberg Retina Tomograph. Ophthalmology 2002; 109: 1072-1077.

18 Rhee DJ, Greenfield DS, Chen PP, Schiffman J. Reproducibility of retinal nerve fiber layer thickness measurements using scanning laser polarimetry in pseudophakic eyes. Ophthalmic Surg Lasers 2002; 33: 117-122. 